\title{
Lateral plantar nerve injury following steroid injection for plantar fasciitis
}

\author{
D M Snow, J Reading, R Dalal
}

Br J Sports Med 2005;39:e41 (http://www.bjsportmed.com/cgi/content/full/39/12/e41). doi: 10.1136/bjsm.2004.016428

A 41 year old man presented with pain and numbness affecting the lateral aspect of his foot after a steroid injection for plantar fasciitis. Examination confirmed numbness and motor impairment of the lateral plantar nerve. The findings were confirmed by electromyographic studies. The anatomy of the lateral plantar nerve and correct technique for injection to treat plantar fasciitis are discussed.

$\mathrm{T}$ he lateral plantar nerve with the medial plantar nerve forms the two terminal divisions of the tibial nerve under the middle of the flexor retinaculum. The lateral plantar nerve crosses the sole obliquely medial to the lateral plantar artery. It supplies flexor accessories and abductor digiti minimi and sends perforating branches through the plantar aponeurosis to supply the skin on the lateral side of the sole. Near the base of the 5th metatarsal bone, it divides into superficial and deep branches (fig l). The superficial branch supplies the 4th cleft and communicates with the medial plantar nerve and, by a lateral branch, supplies the skin of the lateral side and dorsum of the little toe. The superficial branch supplies three muscles, namely the flexor digiti minimi brevis and the two interossei of the 4th space. The deep branch lies within the concavity of the plantar arch and ends by sinking into the deep surface of the oblique head of adductor hallucis. It gives off branches of the remaining interossei, to the transverse head of adductor hallucis and to the three lateral lumbricals. The first branch of the lateral plantar nerve innervates the region of the medial calcaneal tuberosity, the site of maximal tenderness in plantar fasciitis.

\section{CASE HISTORY}

A 41 year old Iranian man originally presented in August 1999 complaining of pain in his heel consistent with plantar fasciitis. He was prescribed a sorbithane heel cup, and $40 \mathrm{mg}$ Depo-medrone/lignocaine was injected using a medial approach. Over the next three months, the symptoms failed to settle and in fact deteriorated. The patient also complained of numbness in the 3rd, 4th, and 5th toes associated with pain on walking. Examination confirmed the presence of numbness but there was no motor deficit.

Nerve conduction studies showed that the lateral plantar sensory nerve action potential was absent on the left but well reproduced on the right. The findings were in keeping with a poorly functioning left lateral plantar nerve and would fit with a history of nerve damage.

We reviewed the patient four years after the initial consultation. The symptoms were unchanged, and on examination there was hypoesthesia along the lateral two thirds of the foot, with motor weakness of toe flexion in the 3 rd, 4th, and 5th toes.

\section{DISCUSSION}

An injection should be made from the medial side after careful localisation of the point of maximal tenderness. The needle is inserted tangentially through the softer skin, so that its point is under the point of maximum tenderness. Corticosteroid injection for the treatment of plantar fasciitis has been advocated by a number of authors. ${ }^{1-3}$ The site of injection depends on the site of pain, although the most common site is the medial calcaneal tuberosity. Reported complications include fat pad atrophy, ${ }^{4}$ rupture of the plantar fascia, ${ }^{5}$ and calcaneal osteomyelitis. ${ }^{6}$ Lateral plantar nerve palsy has never been documented.
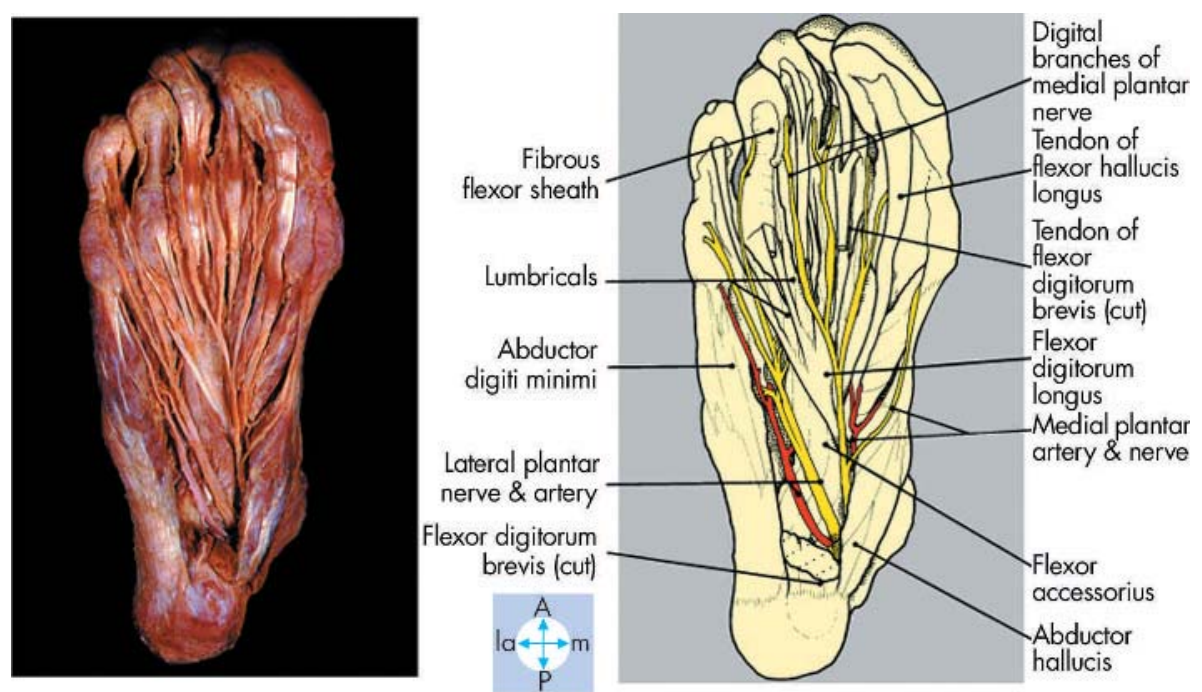

Figure 1 Anatomy of the foot. 
This case shows that injury to the nerve is possible, and probably secondary to intraneural injection. The consequences of such a complication are considerable, with disabling pain and hypoesthesia.

Close attention must be paid to injection technique. Practitioners who carry out injections must have good knowledge of the anatomy of the lateral plantar nerve, and should be vigilant for any evidence of neurological symptoms during the injection. Although this complication is uncommon, patients should be counselled about its possibility before an injection, especially when the site of injection is located over the medial tuberosity.

\section{CONCLUSIONS}

Lateral plantar nerve injury is a potential complication of steroid injection for plantar fasciitis. It can be avoided if close attention is paid to the anatomy of the nerve and injection technique.

\section{Authors' affiliations}

M D Snow, J Reading, R Dalal, Stepping Hill Hospital, Stockport, UK

Competing interests: none declared

Correspondence to: Mr Snow, Orthopaedics, Stepping Hill Hospital, Stockport, UK; snowmartyn@hotmail.com

\section{REFERENCES}

1 Dasgupta B, Bowles J. Scintigraphic localisation of steroid injection site in plantar fasciitis. Lancet 1995;346:1400-1.

2 Lapidus PW, Guidotti FP. Painful heel: report of 323 patients with 364 painful heels. Clin Orthop 1987:39:178.

3 Wolgin M, Cook C, Graham C, et al. Conservative treatment of plantar heel pain: long term follow-up. Foot Ankle 1994;15:97-102.

4 Sellman JR. Plantar fascia rupture associated with corticosteroid injection. Foot Ankle Int 1994:376-81.

5 Acevedo JI, Beskin JL. Complications of plantar fascia rupture associated with corticosteroid injection. Foot Ankle Int 1998;19:91-7.

6 Gidumal R, Evanski P. Calcaneal osteomyelitis following steroid injection: a case report. Foot Ankle 1985;6:44-6.

\section{COMMENTARY}

This paper highlights one of the potential complications of a steroid injection that might otherwise be thought of as a benign procedure. The effects of a steroid injection are generally temporary at best.

M A Wolgin San Diego, CA, USA; wolgin@hotmail.com 